\title{
Photodecomposition and Phototoxicity of Natural Retinoids
}

\author{
William H. Tolleson ${ }^{1}$, Shui-Hui Cherng ${ }^{1}$, Qingsu Xia ${ }^{1}$, Mary Boudreau ${ }^{1}$, Jun Jie Yin ${ }^{2}$, Wayne G. Wamer ${ }^{2}$, Paul C. \\ Howard ${ }^{1}$, Hongtao $\mathrm{Yu}^{3}$, and Peter P. Fu ${ }^{*}$ \\ ${ }^{1}$ National Center for Toxicological Research, U.S. Food and Drug Administration, Jefferson, AR 72079, USA \\ ${ }^{2}$ Office of Cosmetics and Colors, Center for Food Safety and Applied Nutrition, U.S. Food and Drug Administration, College \\ Park, MD 20740, USA \\ ${ }^{3}$ Department of Chemistry, Jackson State University, Jackson, MS 39217, USA \\ *Correspondence to Dr. Peter P. Fu. E-mail: pfu@nctr.fda.gov
}

Received: 15 November 2004 / Accepted: 06 February 2005 / Published: 30 April 2005

\begin{abstract}
Sunlight is a known human carcinogen. Many cosmetics contain retinoid-based compounds, such as retinyl palmitate (RP), either to protect the skin or to stimulate skin responses that will correct skin damaged by sunlight. However, little is known about the photodecomposition of some retinoids and the toxicity of these retinoids and their sunlight-induced photodecomposition products on skin. Thus, studies are required to test whether topical application of retinoids enhances the phototoxicity and photocarcinogenicity of sunlight and UV light. Mechanistic studies are needed to provide insight into the disposition of retinoids in vitro and on the skin, and to test thoroughly whether genotoxic damage by UV-induced radicals may participate in any toxicity of topically applied retinoids in the presence of UV light. This paper reports the update information and our experimental results on photostability, photoreactions, and phototoxicity of the natural retinoids including retinol (ROH), retinal, retinoid acid (RA), retinyl acetate, and RP (Figure 1).
\end{abstract}

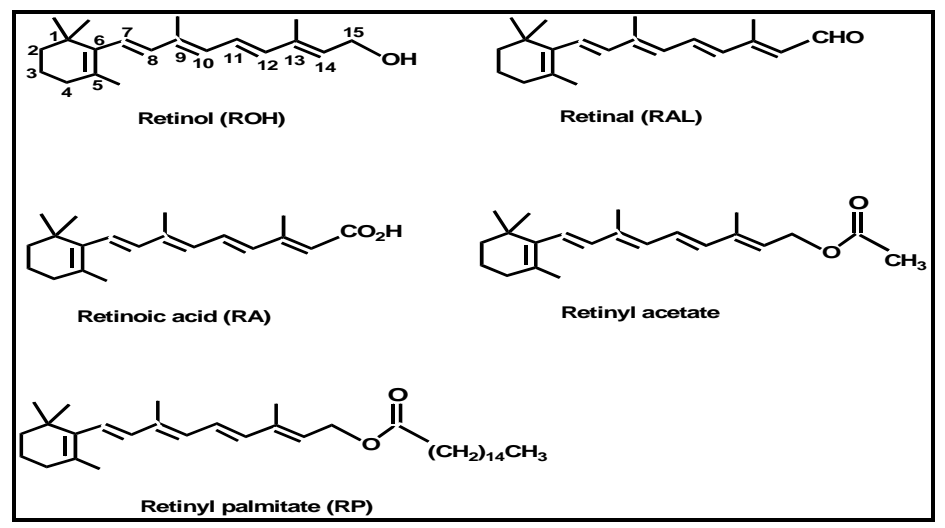

Figure 1: Names and numbering of retinoids.

Keywords: Retinol, vitamin A, retinoic acid, retinyl palmitate, phototoxicity, photocarcinogenicity

\section{Introduction}

Sunlight is a complete carcinogen and is responsible for the induction of squamous cell and basal cell carcinomas in humans (1). Sunlight radiation is a continuum of wavelengths divided into five regions: (i) infrared (above $800 \mathrm{~nm}$ ); (ii) visible (between 400 to 800 $\mathrm{nm}$ ); (iii) UVA (between 315 to $400 \mathrm{~nm}$ ); (iv) UVB (between 280 to $315 \mathrm{~nm}$ ); and (v) UVC (between 200 to $280 \mathrm{~nm})$. Humans are not exposed to UVC light due to stratospheric absorption of UVC by ozone.

Vitamin A (all-trans-retinol; retinol) $(\mathrm{ROH})$ is an important regulator in epidermal cell growth, normal cell differentiation, and cell maintenance (2). It also affects vision, reproduction, morphogenesis, and pattern formation. Metabolism is required to convert retinol to 
biologically active metabolites, e.g., all-trans-retinoic acid (RA, tretinoin) and its cis-isomers (3). Retinyl palmitate (RP) is the principal storage form of retinol in humans and animals and can be enzymatically hydrolyzed back to retinol in vivo. Both RA and its 9-cis isomer exhibit very high biological activities, including inducing epidermal growth and differentiation.

Because the ester forms of retinol are thermally more stable than retinol, both PR and retinyl acetate have been commonly used in cosmetic products. As such, the number of cosmetic products containing RP has increased rapidly in the last two decades. Data available from FDA's Voluntary Cosmetics Registration Program, compiled in accordance with Title 21 Section 720.4 of the Code of Federal Regulations (4), indicate that 102 cosmetic formulations in 1981, 355 cosmetic formulations in 1992, and 667 formulations in 2000 contained RP. Retail product categories containing RP include moisturizing preparations, skin care preparations, night skin care preparations, lipsticks, suntan gels and preparations, makeup preparations, and bath soaps and detergents (5).

\section{Thermal and Photo Stability}

Although RP is thermally more stable than retinol (6), both compounds are still thermally unstable. As shown in Figure 2, the experimental results from our laboratories indicated that $2 \% \mathrm{RP}$ in an oil-in-water emulsion (cream) decomposed gradually at $4^{\circ} \mathrm{C}$, with about $38 \%$ decomposed on day 7. Also, RP, as are retinoids in general, is chemically unstable and its chemical stability is highly dependent on environmental conditions such as solvent, temperature, and availability of oxygen (7). RP is easily thermally-isomerized to the 13-cis isomer. Thermal isomerization is favored in lipophilic solvents and emulsions containing high compositions of oils (7). Anhydroretinol is one of the major decomposition products of RP (8).

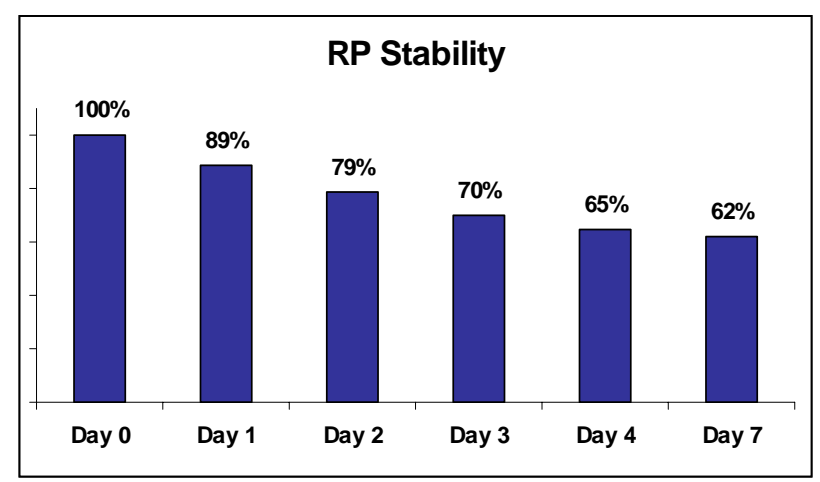

Figure 2: Decomposition of 2\% RP in oil-in-water cream at $4^{\circ} \mathrm{C}$.

It has been shown that RP is much less stable under photoirradiation conditions than is retinol (6). The results shown in Figure 3 confirm that RP (1\% in oil-in-water cream) photodecomposed faster than $\mathrm{ROH}$ under differing doses of UVA radiation. Boehnlein et al. (9) reported that after RP was topically applied in acetone to human skin, about $18 \%$ of RP penetrated the skin in 30 hrs. In addition, approximately $44 \%$ of the absorbed RP was hydrolyzed to retinol by skin esterases. As a result, this suggests that the RP and retinol present during a subsequent irradiation would be subject to photodecomposition. Indeed, we have demonstrated that photoirradiation of RP in ethanol under solar simulated light at an irradiance equivalent to terrestrial sunlight resulted in the formation of multiple photodecomposition products (Figure 4). Furthermore, sunlight-induced photodegradation of retinyl esters proceeds much faster than that of retinol, and it has been suggested that cellular retinol binding protein (CRBP) protects retinol from photodegradation $(9,10)$. Retinyl palmitate (RP) and retinol (ROH) in cream under UVA
irradiation --071403

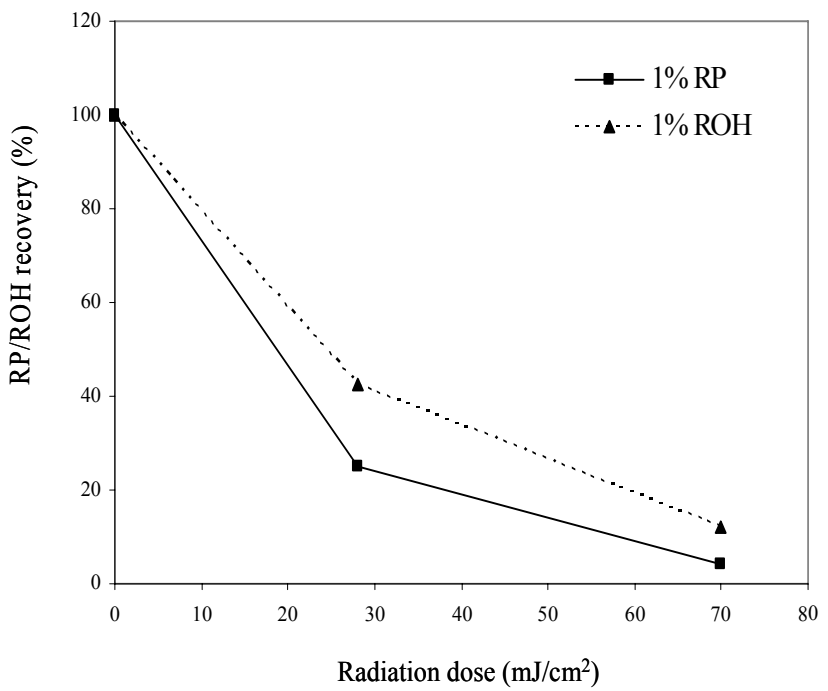

Figure 3: Photodecomposition of RP and $\mathrm{ROH}$.

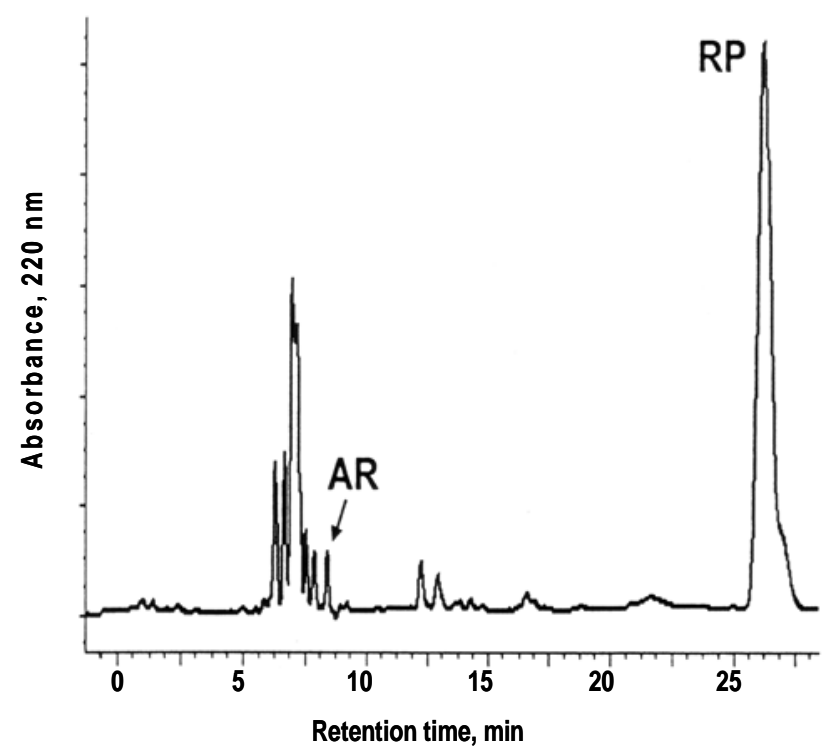

Figure 4: HPLC profile of photodecomposition of RP under sun simulated light $\left(10 \mathrm{~mJ} . \mathrm{CIE} / \mathrm{cm}^{2}\right)$.

\section{Photoreactions}

Photochemical reactions of retinoids proceed through several different routes, including photoisomerization, photopolymerization, photooxidation, and photodegradation (11-13). The types of photodecomposition products that are 
formed are highly dependent on experimental conditions including vehicle, retinoid concentration, dosage and wavelength of the light, photoirradiation time, and the presence of other agents or impurities that can interfere with the photochemical reactions.

\section{Isomerization}

Upon photoirradiation, retinoids including $\mathrm{ROH}, \mathrm{RA}$, $\mathrm{RP}$, and retinyl acetate isomerize into a mixture of transand cis-isomers (14). The solvent can affect both the extent of isomerization of the trans-retinoids and the relative amounts of the different cis-isomers formed (15). All-trans-RA and 13-cis-RA are known to undergo Z-E isomerization and oxidation when exposed to light and air (16). Photodegradation of RA by fluorescent lamps resulted in five isomerization products (17).

During photoirradiation, the primary retinoid photodecomposition products can also isomerize to secondary products. For example, as shown in Figure 4, anhydroretinol (AR) formed from photoirradiation of RP is initially in a trans-form. But during photoirradiation, it isomerizes into a mixture of all-trans-AR and three cis-ARs (Figure 5). The ratio of these isomers is photoirradiation time dependent.
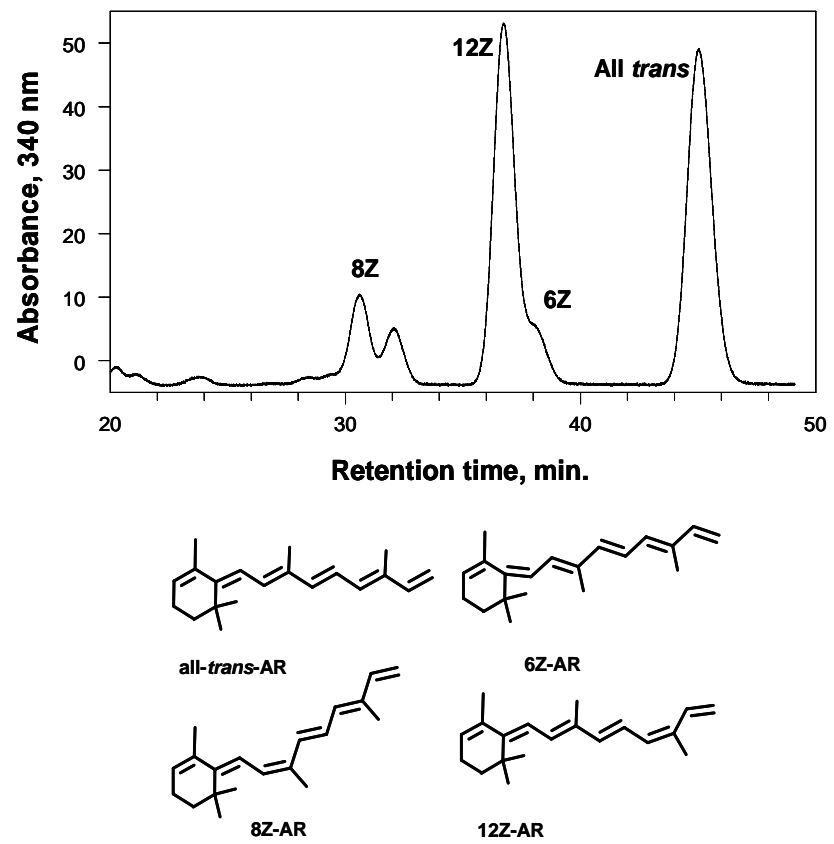

Figure 5: HPLC separation of cis- and trans-isomeric AR. HPLC analysis was conducted on a Vydac $\mathrm{C}_{18}$ column $(4.6 \times 250 \mathrm{~mm})$ eluted isocratically with water in methanol $(\mathrm{v} / \mathrm{v} ; 14 / 86)$ at $1 \mathrm{~mL} / \mathrm{min}$.

Reddy and Rao (18) studied the photoirradiation of retinyl acetate, and showed that isomerization of retinyl acetate and its photodecomposition product (AR) proceeded through an ionic photodissociation mechanism (Figure 6). Upon photoirradiation, retinyl acetate absorbs light energy and results in charge redistribution and change in bond order. Transition into a highly polarized singlet excited state was followed by either isomerization to the cis-retinyl acetate isomers, or release of the acetate anion to form a carbocation.
Isomerization of this carbocation resulted in the formation of the cis-AR isomers.

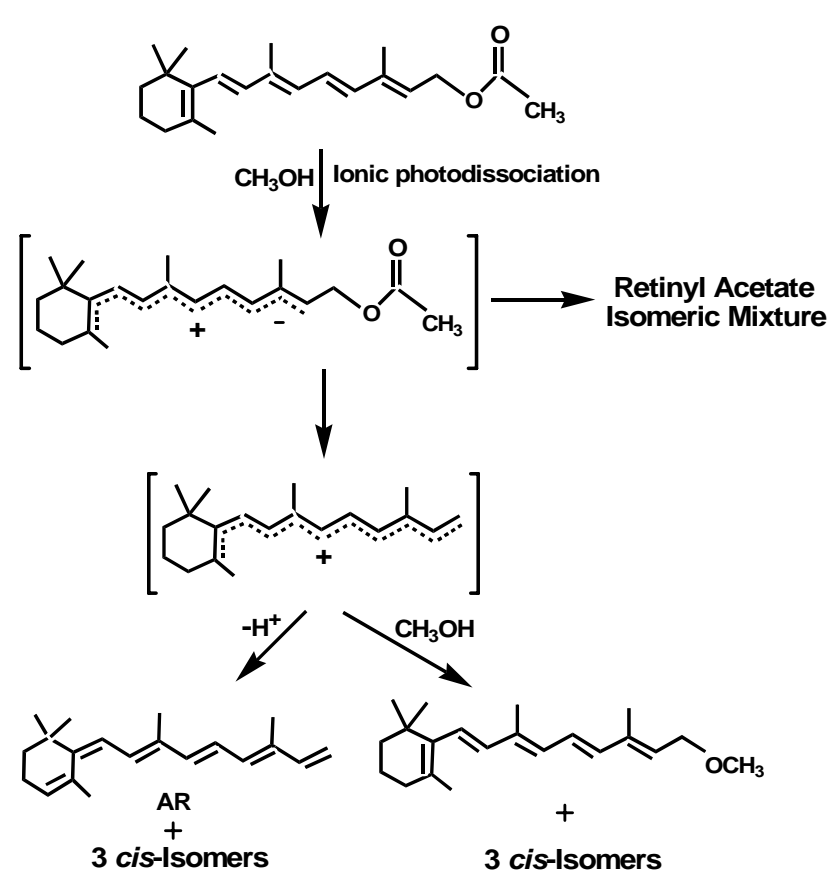

Figure 6: Ionic photodissociation mechanism of isomerization of retinyl acetate under UVA light irradiation to cis-retinyl acetate and a mixture of cis- and trans-AR.

\section{Photoreaction of Retinoids}

Photoreaction of retinoids produces oxidized products, such as ROH 5, 6-epoxide, 4-keto-ROH, and photodecomposition (or photodegradation) products. Photoirradiation of retinol in ethanol by UVC light $(254 \mathrm{~nm})$ resulted in the formation of retinal, $\mathrm{ROH}$ 5,6-epoxide, 5,8-epoxyretinol, and 13,14-epoxyretinol (19). The photooxidation of retinal formed 5,8-endoperoxide (19). Photooxidation of RA in $90 \%$ ethanol resulted in the formation of a number of oxidized products (16). The exposure of RP in methanol to UVA yielded palmitic acid, $\mathrm{AR}$, and 4,5-dihydro-5-methoxyanhydroretinol $(20,21)$. RP irradiated by UVC light $(254 \mathrm{~nm})$ produced AR, palmitic acid, and 2-butenyl palmitate (19). AR was also formed from photoirradiation of RP in ethanol under UVA light (22). Photooxidation of retinyl acetate in benzene with a trace of water produced the non-retinoids dihydroacetinidiolide, 2-hydroxy-2,6,6-trimethylcyclohexanone, $\beta$-ionone, geronic acid and desoxyxanthoxin (23).

It is known that UVA light can photoactivate endogenous and exogenous photosensitizers (19). As expected, photooxidation of retinol and RP in the presence or absence of a photosensitizer resulted in the formation of different products (19). Since skin contains various endogenous photosensitizers and exogenous photosensitizers from topical application, photoreactions of retinoids in the skin are much more complicated than photoreactions performed in simple solutions. 


\section{Mechanisms of Photoreaction}

Besides the ionic photodissociation mechanism described above, photoirradiation of retinoids through free radical mechanism and generation of reactive oxygen species (ROS) has also been reported. In general, Type I photosensitization reactions occur when light is absorbed by a chromophore, and this molecule enters a photoexcited singlet state. It may then undergo intersystem cross-over and form a transient excited triplet state that can interact with other molecules and produce radicals via hydrogen transfer. In the presence of oxygen, superoxide anions may be formed, subsequently generating hydroperoxide radicals or hydrogen peroxide. In the absence of oxygen, radical anions may be formed or photoadditions can occur $(13,24,25)$. Thus, irradiation $(>300 \mathrm{~nm})$ of retinal and retinol in methanol produces free radicals, as does irradiation of RP in dimethylformamide (13). Our study has confirmed that photoirradiation of retinol in $70 \%$ ethanol in water generated free radicals as detected by electron spin resonance (ESR) spectroscopy (Figure 7).

$$
\begin{aligned}
& \text { 4.POBNalone } \\
& \text { with UV on } 30 \text { min }
\end{aligned}
$$
ROH/4OBN
with UV off $\mathrm{ROH} / 4 \mathrm{POBN}$ with

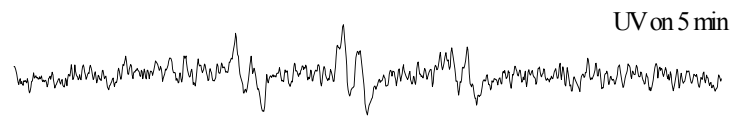

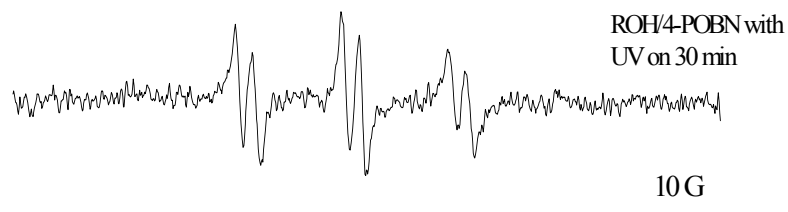

Free radical generation from $\mathrm{ROH}$ under $\mathrm{UV}$ irradiation

Figure 7: ESR measurements of $0.35 \mathrm{mg} / \mathrm{ml} \mathrm{ROH}$ in $70 \%$ ethanol in water containing $200 \mathrm{mM}$ spin trap $\alpha$-(4-Pyridyl-1-oxide)-N-tert-butylnitrone (POBN) after being photoirradiated with UVA light at wavelength 320 nm. Conventional ESR spectra were obtained with a Varian E-109 X-band spectrometer. ESR signals were recorded with $15 \mathrm{~mW}$ incident microwave and $100 \mathrm{kHz}$ field modulation of $1.25 \mathrm{G}$. All measurements were performed at room temperature.

In addition to radicals and ions, ROS are produced following irradiation of retinoids. For example, single oxygen is generated when retinal is illuminated in the presence of oxygen $(26,27)$. ROS can initiate lipid peroxidation and produce lipid alkoxy radicals and several tumorigenic small aldehydes $(28,29)$.

\section{Phototoxicity}

Because they are exposed to the environment, the skin and eyes are more vulnerable to phototoxic damage than other organ systems. Retinoids are naturally abundant in both of these tissues and participate in specific phototoxic mechanisms. Photoirradiation of retinoids may generate acute and chronic toxicity through the formation of photoreaction products that are toxic, or photoexcitation of retinoids forming the excited retinoid species that exert toxicity directly or indirectly (Figure 8). Retinoids absorb light in the UVA range (315-400 nm) and thus would be photoexcited by light containing UVA. For instance, RP has a maximum UV-visible absorption at $326 \mathrm{~nm}$ (3) and thus, may be able to absorb UVA light and act as a photosensitizer. Thus, photoactivation of RP could generate short-lived ROS that have been shown to damage DNA and proteins and lead to tumors.

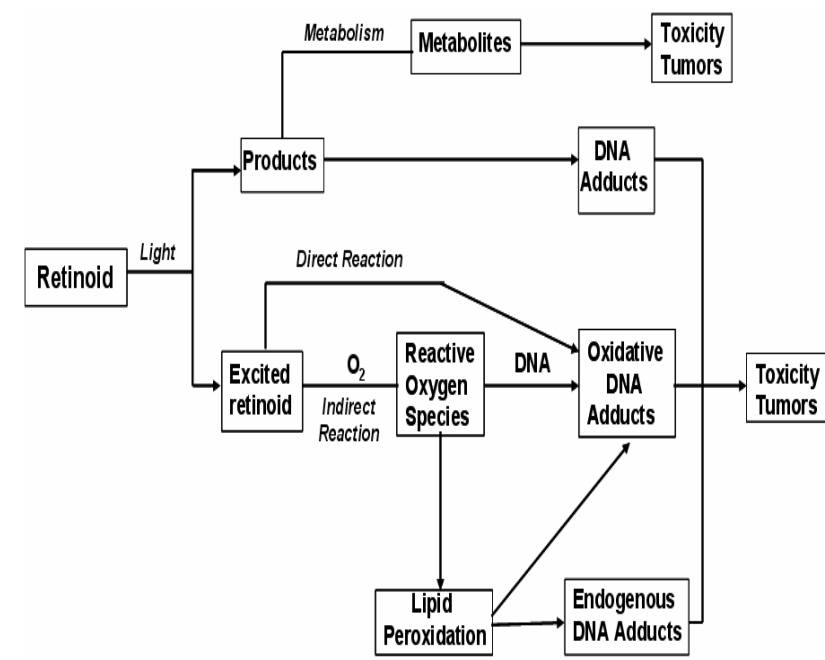

Figure 8: Potential photoreaction pathways of retinoids leading to phototoxicity and tumor formation.

The depth to which solar radiation penetrates tissues, and thereby induces adverse biological effects, depends strongly on its wavelength. In addition, the spectral transmission/absorption characteristics of solar-exposed tissues (Figure 9) influence penetration of light. These and other factors result in the generation of action spectra for photoinjuries to skin and eye components. The action spectra for each photohazard category (Figure 10) were applied to the most sensitive components of the eye or skin to arrive at threshold limit values (TLV) for ocular and cutaneous exposures to monochromatic and broadband light sources. The TLV were established in 1996 by the American Conference of Governmental Industrial Hygenists (ACGIH) (30).

Negligible amounts of UVC are present in solar radiation at sea level and are generally incapable of penetrating the outer cornified layers of the epidermis or the cornea of the eye. However, protective shielding is required to protect the eyes and skin of workers from intense sources of UVC in the workplace, such as electric arc welding. Failure to prevent ocular UVB or UVC exposure leads to photokeratitis of the cornea (31), also known as "welder's burn" and "snow blindness." UVA and UVB penetrate the anterior chamber of the eye where absorption by the lens may induce cataract formation. 

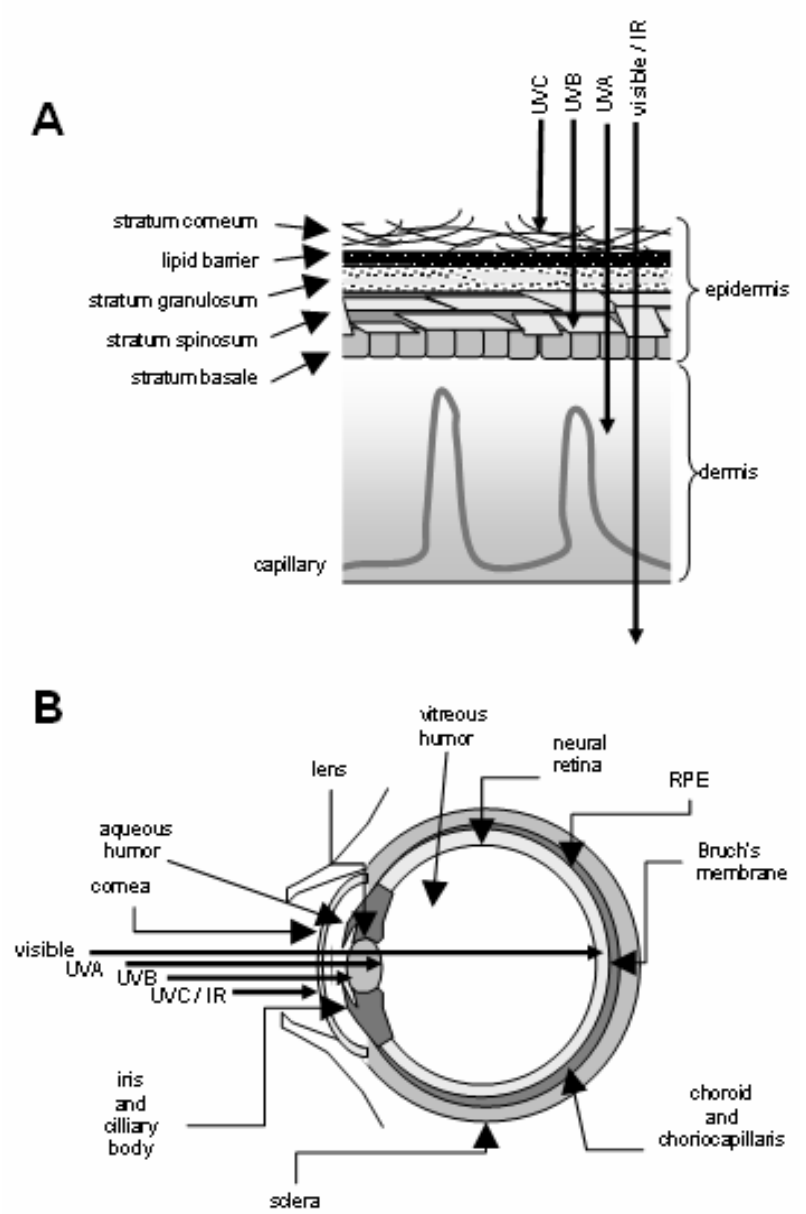

Figure 9: Penetration of skin and eye by solar radiation. (A) Diagram of skin. (B) Diagram of eye.

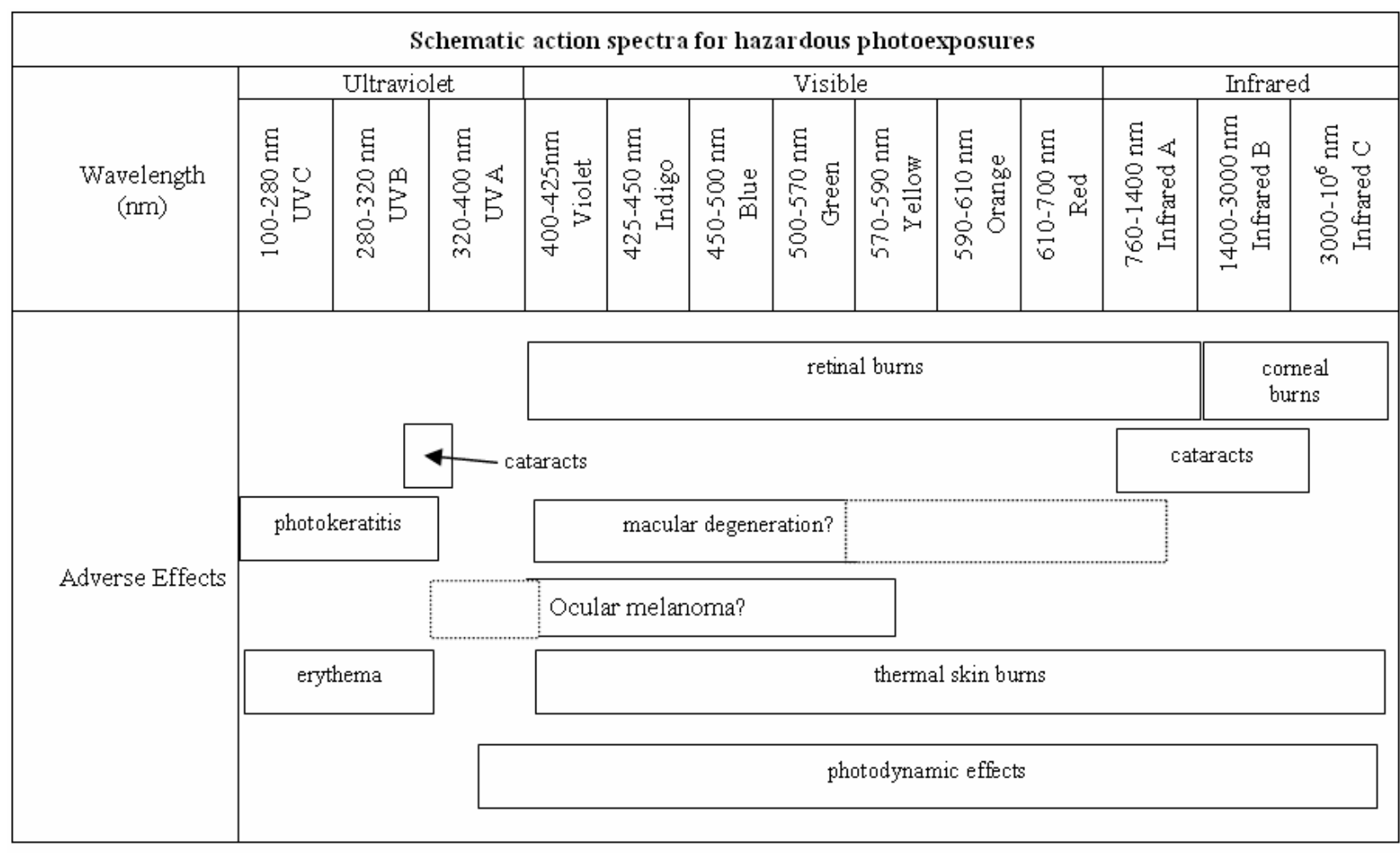

aAdapted from Sliney, J. Photochem. Photobiol., 2001.

Figure 10: Schematic action spectra for hazardous photoexposures (31). 
The anterior layers of the eye are transparent to visible wavelengths but are absorbed by retina and the underlying choroid. Ocular exposure to intense blue light is believed to play a role in age-related macular degeneration and, possibly, ocular melanoma. Infrared radiation is strongly absorbed by the cornea and excessive exposure can cause corneal burns. Solar UVB penetrates the epidermis of nonpigmented skin to cause erythema and skin cancers. The superficial capillary bed of the dermis can be reached by UVA, visible, and infrared radiation and may cause photodynamic effects via absorption by blood-borne chromophores. Cutaneous exposure to visible or infrared wavelengths that penetrate the full depth of normal skin can produce thermal burns.

Phototoxic autofluorescent lipofusin granules, comprised of complex indigestible lipid/protein aggregates, accumulate intracellularly as a consequence of aging. The accumulation of lipofusin in retinal pigment epithelial (RPE) cells is associated with increased photooxidative damage and is believed to contribute to macular degeneration and blindness. The major fluorophore detected in organic extracts of lipofuscin from RPE cells is an unusual pyridinium bisretinoid called A2E (Figure 11) generated as a byproduct from phosphatidylethanolamine and excess all-trans-retinal that evades recycling by RPE cells. Because it cannot be metabolized further, A2E accumulates in RPE lysosomes where it impairs clearance of phospholipids derived from phagocytosed rod outer segments and sensitizes RPE cells for apoptosis. Irradiation with blue light of the lipofuscin organic extracts of RPE cells generates ROS and produces lipid peroxidation. Irradiation of A2E also releases ROS, but the quantum yield is insufficient to account for the amount of ROS produced by lipofuscin. Ocular lipofuscin granules also contain all-trans-retinal which is 73-fold more efficient than A2E at photochemically generating singlet oxygen and 3.2-fold more efficient at generating superoxide radicals.

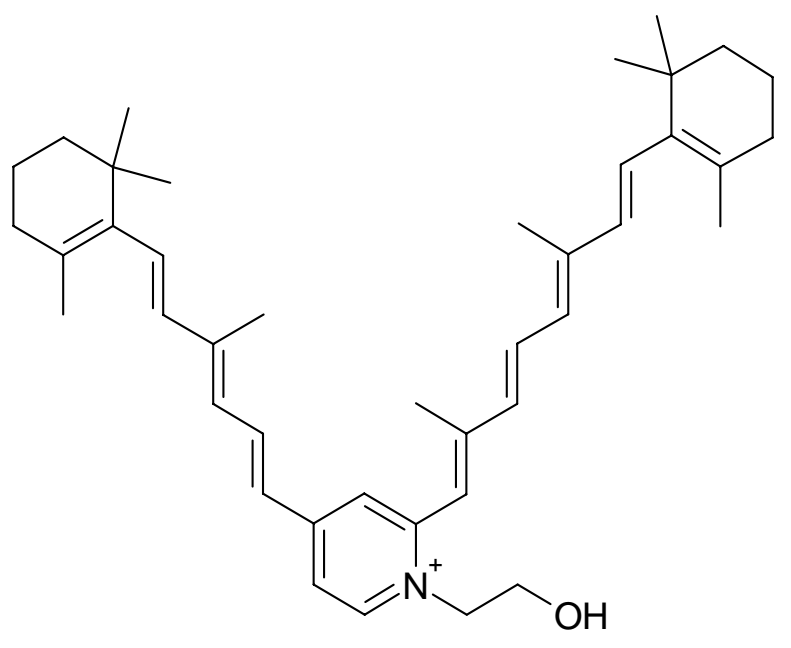

A2E (all-trans)

N-retinylidene-N-retinylethanolamine

Figure 11: A2E, a fluorescent retinoid derivative isolated from retinal pigment epithelial cells

\section{Cytotoxicity and Genotoxicity}

Photoirradiation of RP by UVA light generates ROS, induces lipid peroxidation and causes DNA single strand cleavage in supercoiled $\Phi$ X174 plasmid DNA $(32,33)$. The phototoxicity of RP in human skin Jurkat T-cells also leads to cytotoxicity and DNA damage (33). Irradiating A2E with $21.6 \mathrm{~J} / \mathrm{cm}^{2} 430 \mathrm{~nm}$ light in PBS generated several derivatives containing 1-7 epoxide groups per A2E molecule (34). Cultured human RPE cells treated with A2E epoxides exhibited DNA damage detected by using the single cell gel electrophoresis/COMET assay.

\section{Effect on Gene Expression}

Certain naturally occurring retinoids influence gene expression directly by acting as agonists (e.g., RA) or antagonists (e.g. AR) for retinoic acid or retinoid-x receptors that regulate the transcription of genes containing functional retinoic acid response elements (RAREs). The expression of ROS-responsive genes may also be affected by retinoids acting either as antioxidants or as pro-oxidants via photochemical generation of ROS.

UV radiation also influences gene expression by several mechanisms (see reference 35 for review). The release of latent growth factors (EGF, TGF- $\beta$ ) or cytokines (TNF- $\alpha$ ) by UV exposure can stimulate their cognate receptors in an autocrine or paracrine manner. UV exposure can also trigger the release of lipid signaling molecules such as prostaglandin E2, platelet activating factor, ceramide, and lysophosphatidylcholine. Ligand-independent stimulation of receptors (EGFR, KGFR, Fas, TNFR, and TGF $\beta$ R) and activation of downstream signal transduction pathways (ras, PKC, PLC $\gamma, \operatorname{Smad} 2 / 3$ ) can be caused by UV radiation. The classic cellular "UV response" involves transcriptional activation of immediate early genes c-fos and c-jun.and activation of the transcription factors NF- $k$ B and AP- 1 . MAP kinase family members Mek-1/2, ERK-1/2, p38 MAPK, and c-Jun N-terminal kinases (JNKs) are activated by UV radiation and transduce signals to activate various transcription factors including Ets-1. ATF-3, a member of the CREB/ATF family, is strongly induced in cultured fibroblasts by sublethal doses of UVA (36). UVB-induced DNA damage is detected by the ataxia-telangiectasia mutated (ATM) and ataxia-telangiectasia and RAD3-related (ATR) systems. DNA damage signals are transduced via the checkpoint kinases CHK-1 and CHK-2 to p53, activating its ability to regulate the promoters of genes relevant to cell cycle arrest and apoptosis.

The global effects of UV or retinoids on gene expression have been surveyed using DNA microarray technology (37-39). Coexposure to retinoids and UV provides the potential for synergistic or antagonistic effects on gene expression. In our laboratory, we compared the expression of the insulin-like growth factor-1 gene in the skin of Skh-1 hairless mice exposed to suberythemal doses of simulated solar light in the presence or absence of a topical cream containing 13\% RP over 13 weeks (Figure 12). Exposure to simulated solar light or topical RP induced IGF-1 expression, although the IGF-1 promoter lacks a canonical RARE. 
The mechanisms by which RP and SSL influence IGF-1 expression are under investigation.

\section{IGF-1 gene expression}

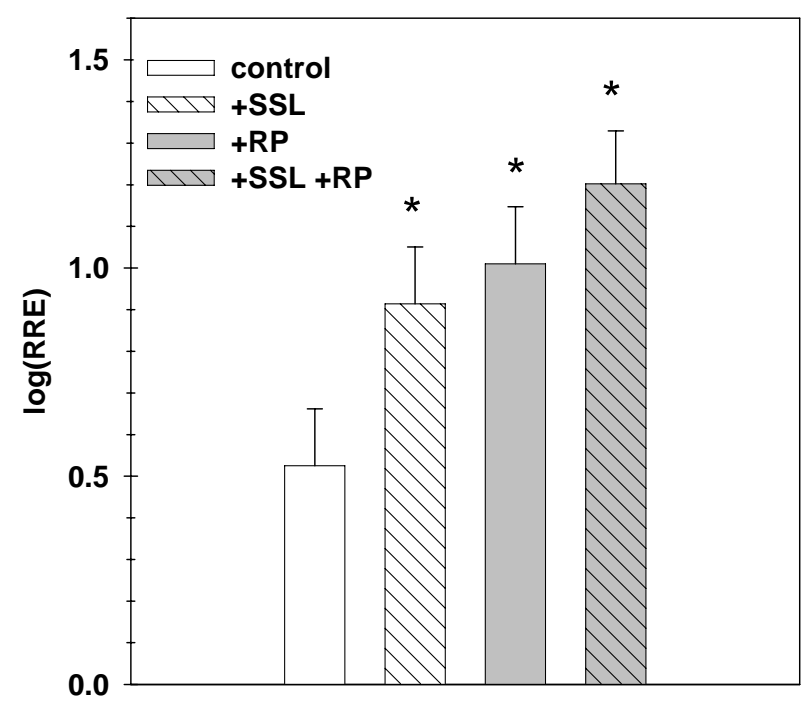

Figure 12: Simulated solar light and retinyl palmitate increase mRNA levels for insulin-like growth factor-1. Groups of HR/Skh-1 hairless mice $(n=13)$ were exposed to simulated solar light (SSL) and/or 13\% retinyl palmitate (RP) three times per week for 13 weeks. Whole skin samples were collected in RNAlater (Ambion) 48 hours after the final treatments. Total RNA was extracted using RNeasy Mini kits (Qiagen). IGF-1 and 18S rRNA levels were determined using qRT-PCR. The relative ratio of expression (RRE) for each group was calculated by the method of Pfaffl (40). Log transformation was used to normalize the data prior to Two Way ANOVA $(p<0.05)$.

\section{Tumorigenicity}

There have been no studies on the effect of topically applied retinol, retinal, RP, and retinyl acetate on the carcinogenicity of UV light. However, retinyl acetate was reported as a co-carcinogen in carcinogenesis studies with butylated hydroxyanisole (BHA) using male F344 rats (41). The effects of topically applied RA on photocarcinogenicity in mice have been investigated by several research groups (42-47). The results are quite varied, with retinoid application increasing, decreasing, or having no effect on photocarcinogenesis. The drastically different effects on tumor incidence can probably be ascribed to differences in study design, particularly the use of different light sources and doses of light radiation. However, the current knowledge of the effects of RA on photocarcinogenesis does not allow a mechanistic explanation for the different outcomes.

\section{Perspective}

Regarding human toxicity, the long-term consequences of using cosmetics containing RP are currently unknown. It has been demonstrated that photoirradiation of RP can result in forming toxic photodecomposition products, generate ROS, induce lipid peroxidation, and cause DNA damage. Also, topically applied RP produces many of the cutaneous changes associated with the use of drug products containing RA which in some instances can enhance photocarcinogenesis. Thus, a study of the photocarcinogenesis of RP, under conditions relevant to the use of RP in cosmetics, is timely and important. As a consequence, RP has recently been nominated by the U.S. FDA and selected by the National Toxicology Program (NTP) as a high priority compound for phototoxicity and photocarcinogenicity studies. The goal of these studies is to provide relevant information necessary for risk assessment of RP in cosmetic creams.

Acknowledgement: We thank Drs. Frederick A. Beland and John Bucher for critical review of this manuscript. This research was supported in part by an Interagency Agreement \#2143-0001 between the Food and Drug Administration/National Center for Toxicological Research (FDA/NCTR) and the National Institute for Environmental Health Sciences/National Toxicology Program (NIEHS/NTP). Through this agreement, this research was supported by an appointment (S.C.) to the Postgraduate Research Program at the NCTR administered by the Oak Ridge Institute for Science and Education through an interagency agreement between the U.S. Department of Energy and the FDA.

\section{References}

1. (IARC): Solar and ultraviolet radiation. IARC Monographs on the Evaluation of Carcinogenic Risks to Humans, International Agency for Research on Cancer, 1992, Vol 55.

2. Idson, B.: Vitamins in cosmetics, an update I. Overview and Vitamin A. Drug Cosmet. Ind., 1990, 146, 26-91.

3. (IARC): Vitamin A. International Agency for Research on Cancer. Handbooks of Cancer Prevention, Lyon, French 1998, Vol 3.

4. Code of Federal Regulations, Washington, D.C. 2000 , Title 21, Part 720.4 (d)(1).

5. The Cosmetic, Toiletry and Fragrance Association, International Cosmetic Ingredient Dictionary and Handbook, Eighth Edition 2000, Eds: Wenninger, J.; Canterbery, R.C. and McEwen, G.N. Jr., CTFA, Washington, D.C., 1999, p. 1279.

6. Ihara, H.; Hashizume, N.; Hirase, N.; Suzue, R.: Esterification makes retinol more labile to photolysis. J. Nutr. Sci. Vitaminol., 1999, 45, 353-358.

7. Ji, H.-G.; Seo, B.: Retinyl palmitate at $5 \%$ in a cream: its stability, efficacy and effect. Cosmet. and Toilet., 1999, 114, 61-68.

8. McBee, J. K.; Kuksa, V.; Alvarez, R.; de Lera, A. R.; Prezhdo, O.; Haeseleer, F.; Sokal, I.; Palczewski, K.: Isomerization of all-trans-retinol to cis-retinols in bovine retinal pigment epithelial cells: dependence on the specificity of retinoid-binding proteins. Biochemistry, 2000, 39, 11370-11380.

9. Boehnlein, J.; Sakr, A.; Lichtin, J. L.; Bronaugh, R. L.: Characterization of esterase and alcohol dehydrogenase activity in skin. Metabolism of retinyl palmitate to retinol (vitamin A) during percutaneous absorption. Pharm. Res., 1994, 11, 1155-1159. 
10. Roos, T. C.; Jugert, F. K.; Merk, H. F.; Bickers, D. B.: Retinold metabolism in the skin. Pharm. Rev., 1998, 50, 315-333.

11. Mousseron-Canet, M.; Mani, J-C.; Favie, C.; Lerner, D.: On the Photochemical Isomerization of Vitamin A. Compt. Rend., 1966, 262, 153-155.

12. Mousseron-Canet, M.: Photochemical Transformation of Vitamin A. "Vitamins and Coenzymes", McCormic. D. B.; Wright, L. D. (Eds.) Methods in Enzymology. 1971, 591-615.

13. Dillon, J.; Gaillard, E. R.; Bilski, P.; Chignell, C. F.; Reszka, K. J.: The photochemistry of the retinoids as studied by steady-state and pulsed methods. Photochem. Photobiol., 1996, 63, 680-685.

14. Fu, P. P.; Cherng, S. -H.; Coop, L.; Xia, Q.; Culp, S. J.; Tolleson, W. H.; Wamer, W. G.; Howard, P. C.: Photoreaction, phototoxicity, and photocarcinogenicity of retinoids. Environ. Carcinogen. Ecotoxicol. Rev., 2003, 21(2), 165-197.

15. Landers, G. M.; Olson, J. A.: Absence of isomerizaiton of retinyl palmitate, retinol, and retinal in chlorinated and nonchlorinated solvents under gold light. J. AOAC. Int., 1986, 69, 50-55.

16. Teraoka. R.; Konishi. Y.; Matsuda. Y.: Photochemical and oxidative degradation of the solid-state tretinoin tocoferil. Chem. Pharm. Bull, 2001, 49, 368-372.

17. Murayama, A.; Suzuki, T.; Matsui, M.: Photoisomerization of retinoic acids under room light: a warning for cell biological study of geometrical isomers of retinoids. J. Nutr. Sci. Vitaminol., 1997, 43, 167-176

18. Reddy, A.M.; Rao, V. J.: Ionic photodissociation of polyenes via a highly polarized singlet excited state. J. Org. Chem., 1992, 57, 6727-6731.

19. Crank, G.; Pardijanto, M. S.: Photooxidations and photosensitized oxidations of vitamin A and its palmitate ester. J. Photochem. Photobiol. A, 1995, 85, 93-100.

20. Lamb, L.E.; Zareba, M.; Plakoudas, S. N.; Sarna, T.; Simon, J. D.: Retinyl palmitate and the blue-light-induced phototoxicity of human ocular lipofuscin. Arch. Biochem. Biophys., 2001, 393, 316-320.

21. Tatariunas. A.; Matsumoto, S.: A retinyl palmitate model of the phenomenon of the intrinsic fluorescence increase in ceroid-lipofuscin cytosomes. Exp. Gerontol., 2000, 35, 1327-1341.

22. Fu, P. P.; Xia, Q.; Blankenship, L. G.; Webb, P. J.; Wamer, W. G.; Howard, P. C.: Photostability and photogenotoxicity of retinyl palmitate. 42nd SOT Annual Meeting, Abstract \#1007, 2003.

23. Isoe, S.; Hyeon, S. B.; Katsumura, S.; Sakan, T.: Photooxygenation of carotenoids. II. The absolute configuation of loliolide and dihydroactinidiolide. Tetrahedron. Lett., 1972, 25, 2517-2520.

24. Boulton, M. E.; Rozanowska, M.; Rozanowska, B.: Retinal photodamage. J. Photochem. Photobiol. B, 2001, 64, 144-161.

25. Ferguson, J.; Johnson, B. E.: Retinoid assoicated phototoxicity and photosensitivity. Pharmac. Ther., 1989, 40, 123-135.

26. Delmelle, M.: Retinal damage by light: possible implication of singlet oxygen. Biophys. Struct. Mech., 1977, 3, 195-198.
27. Delmelle, M.: Possible implication of photooxidation reactions in retinal photo-damage. Photochem. Photobiol., 1979, 29, 713-716.

28. El Ghissassi, F.; Barbin, A.; Nair, J.; Bartsc,h H.: Formation of $1, N^{6}$-ethenodeoxyadenosine and $3, N^{4}$-ethenodeoxycytidine by lipid peroxidation products and nucleic acid bases. Chem. Res. Toxicol., 1995, 8, 278-283.

29. Wang, M. -Y.; Liehr, J. G.: Induction by estrogens of lipid peroxidation and lipid peroxide-derived malondialdehyde-DNA adducts in male Syrian hamsters: role of lipid peroxidation in estrogen-induced kidney carcinogenesis. Carcinogenesis, 1995, 16, 1941-1945.

30. Threshold limit values for chemical substances and physical agents and biological exposure indices, American Conference of Governmental Industrial Hygenists (ACGIH), 1996.

31. Sliney, D. H.: Photoprotection of the eye - UV radiation and sunglasses. J. Photochem. Photobiol., 2001, 64, 166-175.

32. Xia, Q.; Cherng, S.-H.; Blankenship, L. R.; Wamer, W. G.; Bucher, J. R.; Howard, P. C.; Fu, P. P.: Formation of reactive oxygen species and lipid peroxides from photoirradiation of retinyl palmitate and its photodecomposition products. The $32^{\text {nd }}$ Annual Meeting of the American Society for Photobiology, Abstract \#164, 2004.

33. Yan, J.; Cherng, S.-H.; Xia, Q.; Wamer, W. G.; Bucher, J. R.; Howard, P. C.; Yu, H.; Fu, P. P.: Photoclastogenicity of retinyl palmitate and its photodecomposition products. The $32^{\text {nd }}$ Annual Meeting of the American Society for Photobiology, Abstract \#165, 2004.

34. Sparrow, J. R.; Vollmer-Snarr, H. R.; Zhou, J.; Jang, Y. P. Jockusch S, Itagaki, Y.; Nakanishi, K.: A2E-epoxides damage DNA in retinal pigment epithelial cells. Vitamin E and other antioxidants inhibit A2E-epoxide formation. J. Biol. Chem., 2003, 278, 18207-18213.

35. Heck, D. E.; Gerecke, D. R.; Vetrano, A. M.; Laskin, J. D.: Solar ultraviolet radiation as a trigger of cell signal transduction. Toxicol. Appl. Pharmacol., 2004, 195, 288-297.

36. Abe, T.; Oue, N.; Yasui, W.; Ryoji, M.: Rapid and preferential induction of ATF3 transcription in response to low doses of UVA light. Biochem. Biophys. Res. Commun., 2003, 310, 1168-1174.

37. Dazard, J. E.; Gal, H.; Amariglio, N.; Rechavi, G.; Domany, E.; Givol, D.: Genome-wide comparison of human keratinocyte and squamous cell carcinoma responses to UVB irradiation: implications for skin and epithelial cancer. Oncogene, 2003, 22, 2993-3006.

38. Sesto, A.; Navarro, M.; Burslem, F.; Jorcano, J.L.: Analysis of the ultraviolet $B$ response in primary human keratinocytes using oligonucleotide microarrays. Proc. Natl. Acad. Sci. USA., 2002, 99, 2965-9670.

39. Lee, K. H.; Chang, M. Y.; Ahn, J. I.; Yu,, D. H.; Jung, S. S.; Choi, J. H.; Noh, Y. H.; Lee, Y. S.; Ahn, M. J.: Differential gene expression in retinoic acid-induced differentiation of acute promyelocytic leukemia cells, NB4 and HL-60 cells. Biochem. Biophy. Res. Commun., 2002, 296, 1125-1133. 
40. Pfaffl, M. W.: A new mathematical model for relative quantification in real-time RT-PCR. Nucleic. Acis Res., 2001, 29, e45.

41. Hasegawa, R.; Takahashi, M.; Furukawa, F.; Toyoda, K.; Sato, H. and Hayashi, Y.: Co-carcinogenic effect of retinyl acetate on forestomach carcinogenesis of male F344 rats induced with butylated hydroxyanisole. Jpn. J. Cancer Res. (Gann), 1985, 79, 320-328.

42. Kligman, L. H.: Retinoic acid and photocarcinogenesis - a controversy. Photodermatology, 1987, 4, 88-101.

43. Forbes, P. D.; Urbach, F.; Davies, R. E.: Enhancement of experimental photocarcinogenesis by topical retinoic acid. Cancer Lett., 1979, 7, 85-90.
44. Halliday, G. M.; Robertson, B. O.; Barnetson, R. St. C.: Topical retinoic acid enhances, and a dark tan protects, from subedemal solar-simulated photocarcinogenesis. J. Invest. Dermatol., 2000, 114, 923-927.

45. Forbes, P. D.: Photocarcinogenesis: an overview. J. Invest. Dermatol., 1981, 77, 139-143.

46. Epstein, J. H.; Grekin, D. A.: Inhibition of ultraviolet-induced carcinogenesis by all-trans retinoic acid. J. Invest. Dermatol., 1981, 76, 178-180.

47. Kligman, L. H.; Kligman, A. M.: Inhibition of ultraviolet-induced carcinogenesis by all-trans retinoic acid. Arch. Dermatol. Res., 1981, 270, 453-462. 